\title{
The X-ray Diagnosis and Signs Analysis of Occult Elbow Fractures in Children
}

\author{
Shuya $\mathrm{Zhou}^{1}$
}

${ }^{1}$ Qingdao University, 308 Ningxia Road, Qingdao, China

\begin{abstract}
Objective: The purpose of this study was to investigate the $\mathrm{X}$-ray diagnosis and signs analysis of children with occult elbow fractures, so as to make a basis for clinical judgment. Methods: A retrospective analysis was conducted on 60 patients with elbow injury admitted to Qingdao affiliated hospital from June 2017 to November 2018, and no fractures were found in any of the 60 patients during the first X-ray examination. According to the bone conditions, the patients were divided into study group with occult elbow fractures and control group without it, with 30 cases in each group. Results: The author found that the rates of cortical humeral collicular protuberance, cortical depression, positive rate of fat pad, positive rate of humerus front, and trabecular disruption in the study group were significantly higher than those in the control group, and the differences were statistically significant $(\mathrm{P}<$ 0.05). Conclusion: For children with occult elbow fractures, X-ray diagnosis can effectively determine obvious symptoms, which is conductive to clinical judgment and detection, and thus is worthy of clinical promotion and application.
\end{abstract}

Keywords: Children Occult Elbow Fractures, Cortical Bone Bulges, Cortical Depression

\begin{abstract}
Introduction
In clinical medicine, children fracture and elbow joint injury are very common, as children are in the growing season and are pretty active ${ }^{[1]}$. However, such joint injuries are often difficult to detect in children, because the X-ray signs of the patient are often mild, resulting in missed diagnosis. For children in the growth and development period, if occult elbow fractures can not be treated in time, which, to some extent, will negatively influence their health and significantly reduce the life quality of patients. Therefore, it is very necessary to further study the X-ray diagnosis and signs of occult elbow fractures ${ }^{[2]}$. In this study, 60 cases of elbow trauma patients admitted to Qingdao affiliated hospital from June 2017 to November 2018 were selected as research objects for retrospective analysis, aiming to investigate the $\mathrm{X}$-ray diagnosis and sign analysis of children with occult elbow fractures and thus provide a basis for clinical judgment. This study have obtained good results, which was shown in the following sections.
\end{abstract}

\section{Materials and methods \\ Clinical data}

A total of 60 patients with pathologically confirmed elbow injuries were collected from Qingdao affiliated hospital from June 2017 to November 2018, and no fracture was found in any of them on the first X-ray examination. The patients were split into study group and control group, with 30 cases in each group. Further, all the patients in the study group patients were diagnosed as occult elbow fracture, while the patients in the control group patients were diagnosed as normal without occult elbow fracture. In the control group, there were 15 male and 15 female children, and the age of children ranged from 1 to 15 years old, with an average age of $(8.23 \pm 2.31)$ years old. Additionally, the study group had 10 male and 20 female children and their age ranged from 1 to 14 years old, with an average age of $(9.01 \pm 1.21)$ years old. Moreover, all patients underwent $\mathrm{X}$-ray examination due to pain sensation within $7 \mathrm{~h}$ after trauma. In this study, the families of the children were informed of this experiment and signed the informed consent.
Observation methods and judgment criteria
In this study, the X-ray fluoroscopy was performed on the front and side of patients' elbow joints. Then the diagnosis was made in conjunction with X-ray observations and the diagnostic criteria were as follows: (1) When there was a fracture line on the front and side of the joint at the traumatic site of the patient, the patient was diagnosed as fracture or occult fracture. (2) For patients with elbow joint injury who were definitely diagnosed as fracture, X-ray plain film was performed on them 2-3 weeks after fracture was found. If no periosteum reaction was observed, they were diagnosed as occult fracture, otherwise, the patients were fracture. After that, the patients with fracture were excluded, and all normal patients and occult fracture patients were classified into control group and study group respectively, aiming to compare the X-ray signs between these two groups. The main observed signs were changes in bone structure, peripheral soft tissue and positive fat pad in the two groups. Additionally, the criteria for the positive characteristics of fat pad were 
as follows: (1) the thickness of the fat pad at the standard lateral radiograph of the elbow joint was larger than $4.81 \mathrm{~mm},(2)$ the patient's joint flexion and extension activities were limited, (3) there was hematoma or effusion in the elbow joint cavity of patients. When patients met the above three points, they can be diagnosed as fat pad sign positive.

\section{Statistical analysis}

This study was performed using the software SPSS22. $0^{[3]}$. In order to investigate the X-ray diagnosis and signs analysis of children with occult elbow fractures, we used the $t$ test as the metric, which was expressed in relative terms. When $\mathrm{p}$ value was less than 0.05 , the differences between study groups and control groups were statistically significant.

\section{Results \\ The comparison between the $X$-ray signs of two groups}

It was found that the rates of cortical humeral collicular protuberance, cortical depression, positive rate of fat pad, positive rate of humerus front, and trabecular disruption in the study group were significantly higher than those in the control group, and the differences were statistically significant $(\mathrm{P}<0.05)$ (Table 1$)$.

Table 1. The comparison between the X-ray signs of two groups (n, \%).

\begin{tabular}{|c|c|c|c|c|c|c|}
\hline Category & $\begin{array}{l}\text { Sample } \\
\text { size (n) }\end{array}$ & $\begin{array}{l}\text { The rate of cortical humeral } \\
\text { collicular protuberance }\end{array}$ & $\begin{array}{c}\text { The rate of cortical } \\
\text { depression }\end{array}$ & $\begin{array}{l}\text { Positive rate of } \\
\text { fat pad }\end{array}$ & $\begin{array}{l}\text { Positive rate of humerus } \\
\text { front }\end{array}$ & $\begin{array}{l}\text { The rate of } \\
\text { trabecular } \\
\text { disruption }\end{array}$ \\
\hline $\begin{array}{l}\text { Study } \\
\text { group }\end{array}$ & 30 & $22(73.33)$ & 6(20.00) & $30(100.00)$ & $16(53.33)$ & $19(63.33)$ \\
\hline $\begin{array}{l}\text { Control } \\
\text { group }\end{array}$ & 30 & $0(0.00)$ & $0(0.00)$ & $14(46.67)$ & $0(0.00)$ & $0(0.00)$ \\
\hline $\mathrm{t}$ & - & 34.736 & 6.667 & 21.8182 & 21.818 & 27.804 \\
\hline$P$ & - & $<0.05$ & $<0.05$ & $<0.05$ & $<0.05$ & $<0.05$ \\
\hline
\end{tabular}

\section{Discussion}

Because children are naturally lively and active, they tend to often fall or collide in their daily life. Meanwhile, their bones are relatively more fragile than those of adults. Thus, if they are not careful, it is very easy for them to break a bone or have joint injuries. When children have persistent traumatic pain, they should see a doctor as soon as possible to ensure that they can receive timely and effective treatment after fracture. Even though children have a strong ability to recover from fracture quickly, it will cause some unnecessary serious consequences if they have occult elbow fractures. Furthermore, in addition to the slow fracture recovery, serious patients will suffer from deformities or even dysfunction ${ }^{[4]}$.

In the X-ray examination, occult fracture is more difficult to judge relative to fracture, while the fat pad is a common sign of occult fracture. When patients' joints are fractured, their fat pads are often raised by the buildup of blood or fluid, which is often the prominent sign of occult fracture. Further, there is no doubted that other signs in X-rays also play an vital role in occult fracture, such as the rates of cortical humeral collicular protuberance, cortical depression, positive rate of fat pad, positive rate of humerus front, and trabecular disruption. In this study, all above medical indexes in study group were significantly larger than those in control group, indicating those indexes were meaningful in the detection of occult fracture. Therefore, in the clinical judgment of X-ray, all signs should be integrated and analyzed comprehensively, so as to avoid errors in the diagnosis and medical accidents.

\section{Conclusion}

Overall, for the children with occult fracture, X-ray diagnosis can effectively determine the obvious signs, which is conducive to clinical judgment and detection, and thus is worthy of clinical promotion and application.

\section{References}

1. Hu J (2016) X-ray Diagnosis and Sign Analysis of Occult Elbow Fracture in Children, Journal of Imaging Research and Medical Applications, 3(23),4625-4626.

2. Shi S, Li S, and Zhou W (2017) X-ray Diagnosis and Sign Analysis of Occult Elbow Fracture in Children, Journal of Practical Orthopaedics, 15(26),123-124.

3. IBM Corp. Released 2013. IBM SPSS Statistics for Windows, Version 22.0. Armonk, NY: IBM Corp

4. Cui L and Wang X (2017) X-ray Diagnosis and Sign Analysis of Occult Elbow Fracture in Children, Journal of Imaging Research and Medical Applications, 4(11),457-458. 